\section{Population-based visual acuity in the presence of defocus well predicted by classical theory}

\author{
Henk A. Weeber, Kristen A. Featherstone, and \\ Patricia A. Piers \\ AMO Groningen B.V., Van Swietenlaan 5, 9728 NX \\ Groningen, Netherlands
}

\begin{abstract}
According to classical theory, visual acuity (VA) can be modeled using the intersection of the eye's modulation transfer function with a retinal threshold function. To date, there have been limited attempts to validate this methodology by comparing theory with actual measured data. We use the methodology to predict the visual acuity in the presence of defocus of a population of cataract patients implanted with diffractive multifocal intraocular lenses. For the prediction, we used a set of physiological eye models that include chromatic and higher order aberrations. We found that the simulations correlated strongly to the clinical outcomes $\left(R^{2}=0.93\right)$. While the simulated VA of the eye models was systematically $0.05 \log M A R$ units lower (better acuity) than the clinical results, this difference was independent of defocus $(p=0.98)$. These results show that when the simple and straightforward classical theory is applied using physiological eye models, accurate predictions of the VA, and through-focus VA of a population can be made. This method may be suited for predicting visual performance of new cataract and refractive treatments. (๑) 2010 Society of Photo-Optical Instrumentation Engineers. [DOI: 10.1117/1.3475956]
\end{abstract}

Keywords: vision; simulations; aberrations; biomedical optics.

Paper 10083LR received Feb. 19, 2010; revised manuscript received Jul. 3, 2010; accepted for publication Jul. 8, 2010; published online Aug. 10, 2010

Visual acuity (VA) is the spatial resolving power of the visual system. It expresses the angular size of detail that can just be resolved by the observer. The limits of visual acuity are imposed by optical and neural factors or their combination. ${ }^{1}$ Measured using a letter chart, visual acuity is a standard test in almost every ophthalmic practice. According to classical theory, visual acuity can be calculated from the spatial frequency at which the eye's modulation transfer function (MTF) intersects with the retinal threshold function., ${ }^{2,3}$ Here, we apply this method using a set of physiological eye models and compare the results with a set of measurements on a population of cataract patients implanted with multifocal intraocular lenses (IOLs).

Cataract patients implanted with conventional, monofocal intraocular lenses lack the ability to accommodate, and hence, they could benefit from an increased depth of field to see objects at different distances. Multifocal IOLs not only supply

Address all correspondence to: Henk A. Weeber, AMO Groningen B.V., Van Swietenlaan 5, 9728 NX Groningen, Netherlands. Tel: 31-50-5296674; E-mail: henk.weeber@amo.abbott.com two simultaneous focal points, one for seeing distant objects and one for viewing near objects (e.g., reading), but also exhibit a depth of focus, which results in a degree of visual performance at intermediate distances. ${ }^{4,5}$

For this study, we modeled the diffractive Tecnis ${ }^{\circledR}$ Multifocal IOL (Model ZM900, Abbott Medical Optics, Inc.) and compared it with the clinically measured defocus curves, obtained from a group of 11 bilaterally implanted Tecnis Multifocal patients.

The Tecnis Multifocal IOL design has a diffractive relief pattern on the posterior surface, splitting the light in equal amounts of $41 \%$ to the far and near focus, while $18 \%$ of the light is lost in nonfunctional diffractive orders. The diffractive add power for near vision is +4 diopters, which corresponds to about +3 diopters in the spectacle plane. The lens also has an aspheric surface on the anterior side of the optic to reduce spherical aberration. This surface has been described elsewhere. ${ }^{6}$

The clinical measurements were performed with 11 patients bilaterally implanted with the Tecnis Multifocal IOL model ZM900. These patients, ages 52 to 76 years old (mean age $65.8 \pm 7.1$ years), and screened for ocular disease, had natural pupil sizes between 2.5 and $4.0 \mathrm{~mm}$ under photopic lighting conditions. Defocus testing was performed using a self-calibrating, self-illuminating $\left(85 \mathrm{~cd} / \mathrm{m}^{2}\right)$ EDTRS chart placed $4 \mathrm{~m}$ in front of the patient; defocus was induced by placing successive minus trial lenses in 0.5-D increments over the patient's best distance correction; and binocular visual acuity was measured at each defocus position, from zero (best correction) to -5 diopters. The number of letters read correctly were recorded and converted to $\log$ MAR values, ${ }^{7}$ including a mathematical correction for the magnification of the trial lenses ${ }^{8}$ (i.e., $-0.03 \log$ MAR at -5 diopter defocus).

Recently, a set of 46 physiological eye models has been introduced to predict the visual performance in terms of retinal image quality of eyes implanted with IOLs. ${ }^{9}$ These eye models demonstrate wavefront aberrations that are characteristic for a cataract population and were also verified against clinical data of contrast vision. ${ }^{6,9}$ Here, we use the same eye models.

For each individual eye model, the power of the IOL was determined based on the eye length and corneal power, using the SRK/T formula. ${ }^{10}$ A spectacle lens was modeled at $12 \mathrm{~mm}$ in front of the cornea. Eye models were refracted for both spherical and cylindrical power to the closest 0.25 -diopter increment. Defocus testing was simulated as follows: (1) a point source target was placed $4 \mathrm{~m}$ in front of the anterior cornea; (2) the eye models had a fixed physical pupil diameter of $3.0 \mathrm{~mm}$, corresponding to an apparent pupil size of approximately $3.3 \mathrm{~mm}$; (3) defocus was obtained by varying the power of the spectacle lens in 0.25-diopter increments; (4) at each defocus position, the simulated visual acuity was calculated. Simulated visual acuity (sVA) is determined by first calculating the average radial polychromatic modulation transfer function (rMTF) for each eye model with varying levels of defocus. The sVA is calculated from the spatial frequency at which the rMTF intersects with the retinal threshold function. ${ }^{2,3}$ The optical simulations were carried out using

1083-3668/2010/15(4)/040509/3/\$25.00 @ 2010 SPIE 


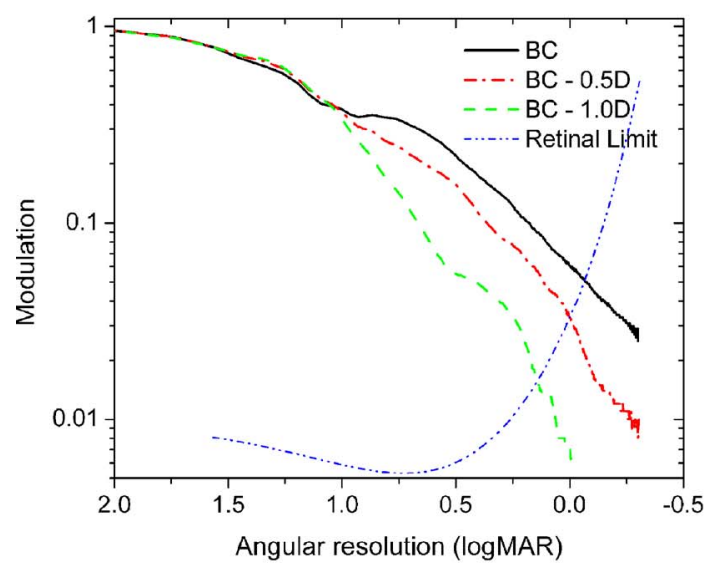

(a)

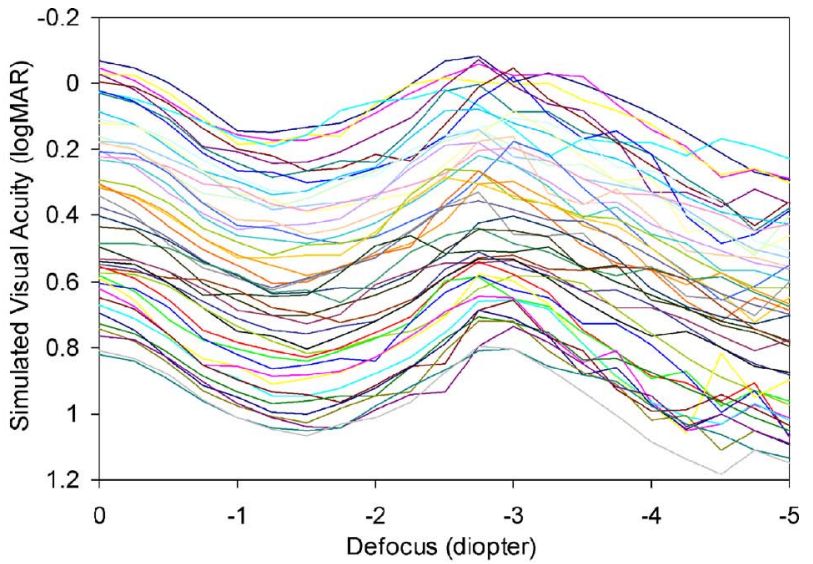

(b)

Fig. 1 (a) rMTF of one of the eye models for the best corrected case (BC) and with -0.5 and -1.0 diopters of (additional) defocus. (b) Resulting defocus curves of 46 eye models, showing the sVA as a function of spectacle defocus. For visualization purposes, each consecutive curve is shifted down by 0.02 logMAR. (Color online only.)

commercial optical design software (OSLO Premium, Lambda Research Corporation).

Figure 1(a) shows an example of the rMTF of one of the eye models, together with the retinal threshold curve. In order to depict the influence of rMTF on spatial vision, the spatial frequency on the horizontal axis is replaced by the angular resolution in logMAR units. The rMTF curve for each defocus value intersects the retinal threshold curve, and this results in the sVA as a function of defocus. These graphs were made for each eye model. Figure 1(b) shows the 46 simulated defocus curves. For visualization purposes, each consecutive curve is shifted down by $0.02 \log$ MAR. All eye models show the same general behavior, having a peak sVA at zero defocus (best spectacle correction) and another peak at a near focus. Figure 2(b) shows the way the clinical defocus curves are usually represented, being an average of the VA results for each defocus value, and with error bars representing the standard deviation. The average defocus curve of the eye models strongly mimics the clinically tested average defocus curve. The graphs show a best near VA at -2.75 diopters defocus (spectacle add) for the eye models, compared to -3.0 diopters for the clinical experiments. It should be noted that in the clinical experiments, the defocus was varied in -0.5-diopter increments, while simulated testing was performed in -0.25 -diopter increments. The best corrected distance sVA was $-0.08 \pm 0.03 \operatorname{logMAR}$, and the best distance corrected

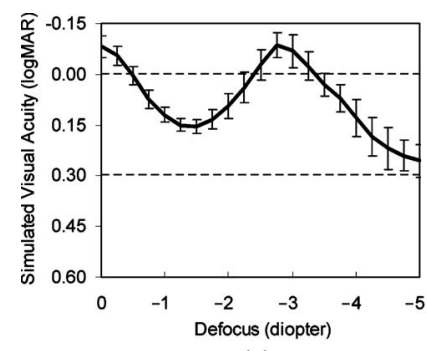

(a)

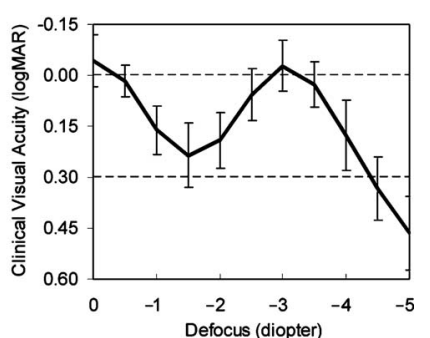

(b)
Fig. 2 Defocus curves obtained from (a) eye model simulations and (b) clinical testing. near sVA was $-0.09 \pm 0.03 \log \mathrm{MAR}$. For the clinical results, these values were $-0.04 \pm 0.08$ and $-0.03 \pm 0.08$, respectively. As generally experienced with a bifocal IOL, there is a reduction in intermediate acuity between the best far and near vision. For the eye models, the lowest average sVA, 0.15 logMAR (20/29 Snellen), is experienced at -1.5 diopters of defocus, meaning a $0.23 \log$ MAR reduction compared to the best focus positions (far and near). Similarly, the clinical results showed that the lowest average VA is at -1.5 diopters of defocus. The average VA at this position was $0.24 \pm 0.09 \log$ MAR (20/34 Snellen), and the reduction compared to the best focus positions (far and near) is 0.27 logMAR units. Within the range of 0 to -4 diopters of defocus, the sVA of the eye models is on average $-0.05 \log$ MAR units lower (better acuity) than the clinical results, and there is no statistically significant relationship between this difference and defocus $(p=0.98)$. The correlation between sVA and clinical VA is shown in Fig. 3. This correlation can be used to fine-tune the predictions of visual acuity using eye models. The figure

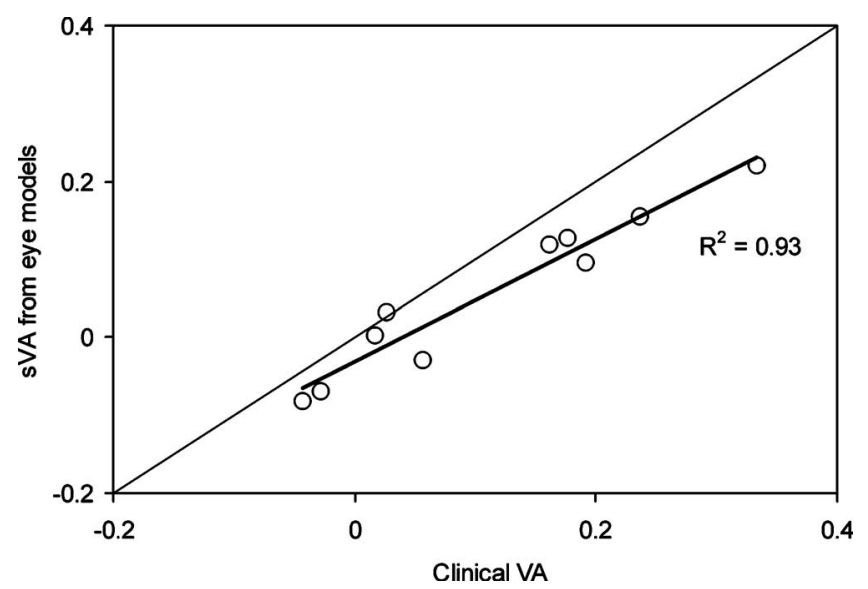

Fig. 3 Plot showing the correlation between the clinically measured visual acuity and the simulated visual acuity from the eye models, both in $\log M A R$, with the range of zero to -4.5 diopters of defocus. Linear regression results in a correlation coefficient of $R^{2}=0.93$. 
shows that the differences between the eye models and clinical results become larger when the VA becomes lower. A possible explanation is that these values are influenced by (lack of) neural adaptation to blur. ${ }^{11}$

The clinical data shows larger standard deviation than the simulations. This can be attributed to intersubject and between-subject variation. Visual acuity measurements have a test-retest variability of about $0.1 \operatorname{logMAR},{ }^{12}$ while simulations have no test-retest variability. In addition, the simulations were not affected by variations in retinal threshold and IOL tilts and decentrations.

While Fig. 2 shows the defocus curves in the way that they are usually presented when reporting clinical results, it should be noted that the curves are affected not only by the VA values, but also by individual differences in patients' defocus value at which the best near VA is achieved. For example, a patient having a best near performance at a large defocus value (e.g., $<-3$ D) may reduce the average intermediate result as well as the average near result. Alternatively, a patient having a best near VA at a small defocus value (e.g., -2 D) may increase the average intermediate VA and decrease the average near VA. To overcome this issue, patient averages can be reported for average best near VA (clinical: $-0.03 \pm 0.07$ $\log$ MAR; eye models: $-0.10 \pm 0.03 \log$ MAR), average defocus at which best near VA occurs (clinical: $-3.09 \pm 0.20$ diopter; eye models: $-2.81 \pm 0.18$ diopter), average lowest intermediate VA (clinical: $0.28 \pm 0.06 \log$ MAR; eye models: $0.16 \pm 0.02 \log$ MAR), and average defocus at which the lowest intermediate performance occurs (clinical: $-1.55 \pm 0.35$ diopter; eye models: $-1.41 \pm 0.19$ diopter). For the clinical data set, these results are very similar to those presented in Fig. 2(b). This indicates that the clinical outcome was very consistent across patients. The impact of averaging defocus curves is affected by the consistency of the clinical procedures of refracting the patients, as well as by the consistency of the population, e.g., in terms of axial length of the eyes. Both factors introduce horizontal variations in the defocus curve.

The eye models were used to simulate the clinical defocus curve testing. The simulated visual acuity was higher than the clinically measured visual acuity. As mentioned earlier, neural adaptation is one of the reasons. Another reason could be choice of the retinal threshold curve, ${ }^{2}$ which was determined under different conditions than those of the clinical test in this study. The simulations used the MTF in the prediction of letter acuity. Letter shapes are complex, however, and letters consist of a range of spatial frequencies and are not rotationally symmetrical, while being compared with a rotationally averaged radial MTF. Also, letters are black-on-white characters having sharp edges, while the MTF represents the modulation of a sine-wave grating. To account for this difference, Greivenkamp et al. ${ }^{3}$ suggested using the square-wave MTF. This approach was not followed in our study, since the retinal threshold function was also based on a sine-wave grating. The simulations did not incorporate the influence of the StilesCrawford effect. However, as the pupils in the eye models are relatively small (physical diameter $3.0 \mathrm{~mm}$ ), the influence of the Stiles-Crawford effect is negligible in the calculation of MTF. $^{13}$

These simulations show that eyes implanted with IOLs that are essentially bifocal still result in functionally useful vision at intermediate distances. This is in contrast with the impression that is obtained when the IOL is tested in an eye model in accordance to the ISO standard for intraocular lenses. ${ }^{14}$ The ISO standard requires IOLs to be tested in a monochromatic, aberration-free eye model. Measurement of the MTF under these conditions shows only the two distinct foci for the multifocal IOL-one for far focus, and one for nearcorresponding to a +4 diopter add power. Between these two foci, there is only measurement noise, which is in sharp contrast to the functional level of visual acuity, as shown in Fig. 2(b). This difference can be attributed to the differences between the ISO standard and the clinical situation as simulated by the eye models, including the use of monochromatic light instead of white light, an aberration-free eye model versus eye models that incorporate residual defocus and astigmatism, spherical aberration and irregular higher order aberration, the inclusion of a retinal limit to seeing detail, and the use of a different optical metric — SVA versus MTF at one specific spatial frequency.

\section{References}

1. A. G. Bennett and R. B. Rabbetts, Clinical Visual Optics, 2nd ed., pp. 19-57, Butterworth-Heinemann, Oxford, UK (1989).

2. F. W. Campbell and D. G. Green, "Optical and retinal factors affecting visual resolution,” J. Physiol. 181(3), 576-593 (1965).

3. J. E. Greivenkamp, J. Schwiegerling, J. M. Miller, and M. D. Mellinger, "Visual acuity modeling using optical raytracing of schematic eyes," Am. J. Ophthalmol. 120(2), 227-240 (1995).

4. S. Cillino, A. Casuccio, F. Di Pace, R. Morreale, F. Pillitteri, G. Cillino, and G. Lodato, "One-year outcomes with new-generation multifocal intraocular lenses," Ophthalmology 115(9), 1508-1516 (2008).

5. A. Artal, S. Marcos, I. Miranda, and M. Ferro, "Through focus image quality of eyes implanted with monofocal and multifocal intraocular lenses," Opt. Eng. 34(3), 772-779 (1995).

6. P. A. Piers, N. E. Norrby, and U. Mester, "Eye models for the prediction of contrast vision in patients with new intraocular lens designs," Opt. Lett. 29(7), 733-735 (2004).

7. F. L. Ferris, III, A. Kassoff, G. H. Bresnick, and I. Bailey, "New visual acuity charts for clinical research," Am. J. Ophthalmol. 94(1), 91-96 (1982).

8. D. A. Atchison and G. Smith, Optics of the Human Eye, ButterworthHeinemann, Oxford, UK (2000).

9. P. A. Piers, H. A. Weeber, P. Artal, and S. Norrby, "Theoretical comparison of aberration-correcting customized and aspheric intraocular lenses," J. Refract. Surg. 23(4), 374-384 (2007).

10. J. A. Retzlaff, D. R. Sanders, and M. C. Kraff, "Development of the SRK/T intraocular lens implant power calculation formula," J. Cataract Refractive Surg. 16(3), 333-340 (1990).

11. S. George and M. Rosenfield, "Blur adaptation and myopia," Optom. Vision Sci. 81(7), 543-547 (2004).

12. D. A. Rosser, I. E. Murdoch, and S. N. Cousens, "The effect of optical defocus on the test-retest variability of visual acuity measurements," Invest. Ophthalmol. Visual Sci. 45(4), 1076-1079 (2004).

13. A. van Meeteren, "Calculations on the optical transfer function of the human eye for white light," Opt. Acta 21(5), 395-412 (1974).

14. International Organization for Standardization (ISO) "Ophthalmic implants-intraocular lenses-part 9. multifocal intraocular lenses," ISO-11979-9 (2006). 